\title{
Cronaca sociale
}

\section{Verbale dell'Assemblea del 29 Marzo 2012}

Ordine del giorno: 1) Comunicazioni della Presidenza, 2) Letture ed approvazione del verbale della precedente Assemblea, 3) Lettura ed approvazione del Bilancio Consuntivo 2011, 4) Lettura ed approvazione Regolamento dei Centri Studi, 5) Ammissione nuovi Soci e Soci dimissionari, 6) Varie ed eventuali.

2) Il Segretario Bellante dà lettura del verbale della precedente Assemblea, compreso il verbale del notaio De Vivo inerente il nuovo Statuto della società. I due verbali vengono approvati all'unanimità.

3) Il Tesoriere Chiozzi illustra il Bilancio Consuntivo 2011, il Revisore dei Conti prof. Laureti, legge la relazione del Collegio dei revisori. Capitolo per capitolo vengono presentate le voci più importanti spiegandone i contenuti. Alla fine dell'anno sociale il bilancio si chiude con un saldo positivo di $€ 2.355,00$.

I Soci presenti in assemblea approvano all'unanimità; i consiglieri non prendono parte alla votazione.

4) Il Presidente Violani presenta il nuovo Regolamento che regola i rapporti tra la SISN e i suoi Centri Studi. Il testo viene approvato dall'Assemblea dei Soci all'unanimità.

5) Il Segretario Bellante presenta l'elenco dei nuovi Soci che chiedono di essere ammessi alla nostra Società. Non essendoci alcun voto contrario da parte dai Soci presenti, la lista viene approvata all'unanimità.

La seduta termina alle ore 19.50 .

Il Segretario

Il Presidente

Claudio Bellante

Carlo Violani

\section{Verbale dell'Assemblea del 18 Dicembre 2012}

Ordine del giorno: 1) Comunicazioni del Presidente, 2) Lettura ed approvazione del verbale della Assemblea precedente, 3) Lettura ed approvazione del Bilancio Preventivo 2013, 4) Ammissione dei nuovi Soci e Soci dimissionari, 5) Varie ed eventuali.

1) Il Presidente apre l'Assemblea alle ore 18,00; comunica che la SISN sta organizzando la giornata di studi in memoria del Professor Cesare Conci, che 
si terrà nell'Aula Magna del Museo il 25 Gennaio 2013. Dà poi la parola al Socio Stefano Aguzzi che presenta lo stato dell'arte sull'organizzazione dell'evento.

2) Il Presidente chiede al Vice-Presidente Barbagli di fungere da verbalizzante, in assenza del Segretario, Barbagli dà lettura del verbale dell'Assemblea precedente che viene approvato all'unanimità.

3) Il Presidente invita il Tesoriere a presentare il Bilancio Preventivo 2013 che viene illustrato in tutte le sue parti. Il Socio Cambieri chiede un chiarimento a cui rispondono i Revisori dei Conti, Teresita Liso e Federico Oriani. Messo ai voti, il Bilancio viene approvato all'unanimità (i Consiglieri non prendono parte alla votazione).

4) Barbagli dà lettura dell'elenco dei Soci che hanno presentato domanda di ammissione alla SISN e di quelli di cui devono essere ratificate le dimissioni. L'Assemblea approva all'unanimità.

Il presidente comunica l'uscita di Atti e Barbagli informa che è in corso la spedizione del numero di RIO recentemente pubblicato. Il Presidente aggiunge che l'ultimo numero di Natura, ha avuto l'onore di una recensione sul Corriere della Sera. Il Socio Veronesi esprime il suo apprezzamento per il medesimo volume.

Il Presidente annuncia l'imminente pubblicazione di un numero di Natura che conterrà gli atti di un convegno sull'esplorazione zoologica dell'Africa, organizzato dal Museo di Storia Naturale di Pavia. Anna Alessandrello informa in merito alla prossima uscita su Memorie della ristampa anastatica dell'opera di Sordelli e Jan sugli Ofidi, curata da Stefano Scali.

Il Socio Spinello prende infine la parola per illustrare le iniziative su Paolo Magretti organizzate dal Comune di Paderno Dugnano (MI), con il patrocinio della SISN.

Il Presidente e tutto il Consiglio augurano ai Soci un felice Natale e un prospero 2013.

Null'altro essendovi da deliberare, l'Assemblea è sciolta alle ore 19,00.

Il Verbalizzante

Fausto Barbagli

Il Presidente Carlo Violani

\section{Attività dei Centri Studi}

\section{Centro Studi di Esobiologia}

Coordinatore: Daniele Venturoli

Nato nel 2001 il Centro Studi di Esobiologia (CSE) si propone come strumento di divulgazione e di ricerca per una disciplina, l'esobiologia, che si trova a cavallo tra astronomia e biologia, ma anche tra planetologia e chimica.

Le scoperte degli ultimi anni, in particolare nel campo dei pianeti extrasolari (cioè orbitanti attorno a stelle diverse dal Sole) e delle molecole organiche rilevate nelle nubi interstellari, negli asteroidi e nelle comete, hanno reso plausibile la possibilità che alla nascita della vita sulla Terra abbia contribuito materiale proveniente dallo spazio. D'altra parte, questo genere di ricerche crea a volte 
aspettative eccessive nei mezzi di comunicazione di massa e interpretazioni forzate da parte del pubblico non specialistico. Il CSE persegue quindi l'obiettivo di divulgare in modo competente e neutrale le attuali conoscenze della scienza riguardo alla nascita della vita sulla Terra e delle condizioni che l'hanno resa possibile, confrontando tali dati con le situazioni che è possibile trovare al di fuori del sistema solare.

Fino dalla sua fondazione, il CSE ha promosso workshop e convegni, e ha portato in luoghi diversi (scuole, circoli culturali, università canoniche e della terza età ecc.) conferenze tenute dai suoi membri e, occasionalmente, da specialisti invitati. Inoltre, alcuni membri del CSE svolgono attività di ricerca, pubblicano su riviste specialistiche e partecipano a congressi nazionali e internazionali. Infine, tre dei fondatori del CSE, Luigi Bignami, Gianluca Ranzini e Daniele Venturoli hanno pubblicato alcuni anni or sono un testo che è ancora un riferimento in questo campo: "La vita nell'universo" (Bruno Mondadori, Milano).

Il coordinatore del CSE è il dr. Daniele Venturoli, che ha prima studiato e lavorato per alcuni anni al Dipartimento di Fisiologia Umana dell'Università Statale di Milano e ha quindi collaborato come biofisico per oltre un decennio con l'Università di Lund, in Svezia. Oggi si dedica invece a tempo pieno alla divulgazione scientifica.

Attività svolte nel 2012

12 aprile: all'Università della terza età (UniTre) di Varedo, Gianluca Ranzini, segretario del CSE, ha tenuto la conferenza "Gravità zero: un volo parabolico a bordo dell'aereo dell'Agenzia Spaziale Europea”.

18 aprile: sempre nell'ambito delle attività che si volgono all'UniTre di Varedo, Daniele Venturoli ha parlato di "Zoo spaziale: animali astronauti".

17 maggio: all'Università del Tempo Libero "Il Centro" di Desio, Daniele Venturoli ha tenuto una conferenza dal titolo: "Ipotesi sull'origine della vita sul pianeta Terra", cui è seguita una tavola rotonda con lo stesso Venturoli, il biochimico Carlo Bosio e monsignor Elio Burlon, moderata da Marco Sanvito, docente di astronomia all’Università del Tempo Libero di Desio.

\section{Centro Studi Faunistica dei Vertebrati \\ Coordinatore: Carlo Biancardi}

Il Centro Studi Faunistica dei Vertebrati (CSFV) si è costituito alla fine del 1997. Finalità del Centro Studi è promuovere e coordinare attività di studio sui Vertebrati, attraverso contatti fra istituzioni scientifiche, ricercatori e studenti, con la costituzione anche di gruppi di lavoro per la realizzazione di progetti di ricerca o tesi di laurea. Prioritaria, in questo quadro, la collaborazione con la sezione di Zoologia dei Vertebrati del Museo Civico di Storia Naturale di Milano e la Direzione del Museo stesso.

Rientrano negli scopi del Centro Studi anche le attività volte a favorire l'incontro fra ricercatori, studenti e semplici appassionati, attraverso l'organizzazione di conferenze, incontri-dibattito o serate di studio e discussione riguardanti vari aspetti della zoologia dei Vertebrati o delle Scienze Naturali in genere. 
Partecipazione a Congressi

Nel corso del 2012 diversi membri del CSFV hanno partecipato alle seguenti iniziative:

VIII Congresso Nazionale di Teriologia (Urban Center, Piacenza, 9-11 maggio 2012). Anna Rita Di Cerbo e Carlo Biancardi hanno presentato un poster dal titolo "Habitat fragmentation and the importance of riparian corridors for mammals: a case study in the Northern Adda Regional Park (SCI IT2030005 Palude di Brivio, Lombardy, Northern Italy)".

IX Congresso Nazionale della Socìetas Herpetologica Italica (Bari - Conversano, 26-30 settembre 2012). Anna Rita Di Cerbo e Carlo Biancardi hanno presentato due poster intitolati "Herpetofauna of the Northern Adda Regional Park (SCI IT2030005 Palude Di Brivio, Lombardy, Northern Italy): management and conservation issues" e "Ecological notes on the Bullfrog Lithobates catesbeianus in an area of the Po plain (Emilia-Romagna, Northern Italy)". Dalila Giacobbe e Salvatore Restivo hanno presentato un poster dal titolo "Analisi territoriale del rapporto Uomo-Rettile attraverso l'elaborazione dei dati del Centro Recupero Fauna Selvatica di Messina", realizzato in collaborazione con il C.R.F.S. di Messina.

XXII Congresso Associazione Nazionale Musei Scientifici (Firenze, 14-16 novembre 2012). Dalila Giacobbe e Salvatore Restivo hanno presentato due poster intitolati "Il ruolo della collezione naturalistica del Distaccamento Forestale di Colle San Rizzo (Messina) nella diffusione della cultura scientifica" e "Attività di studio e valorizzazione della collezione di Mammiferi del Museo Zoologico 'Cambrìa' di Messina".

\section{Tesi di Laurea}

Tesi sperimentale del Corso di Laurea triennale in Scienze Naturali dal titolo Check-list e distribuzione dell'avifauna acquatica nella Riserva Naturale Orientata "Laghetti di Marinello" (Messina, Sicilia); candidato Francesco Rando, Relatore Giuseppe Lo Paro (Università di Messina), Correlatori Dalila Giacobbe (CSFV) e Salvatore Restivo (CSFV).

Divulgazione scientifica

Il CSFV ha inoltre organizzato, singolarmente o in collaborazione con altre associazioni ed enti, le seguenti iniziative di divulgazione scientifica:

26 febbraio - 4 marzo 2012: il CSFV ha partecipato alla manifestazione divulgativa "Attività sui Monti Peloritani per la salvaguardia della natura" realizzata nell'ambito della $4^{\circ}$ Notte della cultura di Messina, in collaborazione con Azienda Foreste Demaniali di Messina, Corpo Forestale Regione Siciliana, Società Italiana di Scienze Naturali, MAN e AOM onlus. I membri del CSFV Salvatore Restivo e Dalila Giacobbe hanno allestito una mostra tematica di animali tassidermizzati e reperti legati al mondo della caccia illegale dal titolo "La Rete del bracconaggio". La mostra è stata concepita con lo scopo di sensibilizzare la popolazione sul tema della lotta al bracconaggio condotta nell'area dello Stretto di Messina dal Corpo Forestale della Regione Siciliana.

27 Ottobre: Il CSFV ha partecipato alla manifestazione "Immersi nella Biodiversità - dai Monti Peloritani ai fondali dello Stretto di Messina" organizzata dall'Acquario di Messina (CNR - CeSPOM) in collaborazione con Azienda Regionale Foreste Demaniali, Società Italiana di Scienze Naturali e AOM onlus, presso le strutture del'Acquario stesso. I membri Dalila Giacobbe e Salvatore Restivo hanno 
tenuto una conferenza dal titolo "Alla scoperta degli uccelli acquatici della Laguna di Capo Peloro", incentrata sull'importanza ecologica della RNO Capo Peloro e sulle specie di avifauna acquatica che vi è possibile osservare durante i diversi periodi dell'anno (foto). Inoltre sono stati esposti alcuni poster presentati dal CSFV durante i congressi CIO 2011 e SHI 2012, con l'intento di promuovere le attività scientifiche del Centro Studi e della SISN. La manifestazione rientra nell'ambito dell'evento nazionale "Biodiversamente" promosso da ANMS e WWF.

\section{Ricerche e progetti}

Anche quest'anno è continuato il "Monitoraggio dell'avifauna acquatica nella R.N.O. "Laguna di Capo Peloro" (Sicilia nord-orientale)". Il progetto a lungo termine del CSFV, condotto da Dalila Giacobbe e Salvatore Restivo, prevede il monitoraggio dell'avifauna acquatica stanziale e che sverna o sosta durante la migrazione nella Riserva Naturale Orientata "Laguna di Capo Peloro". Il sito è rappresentato da un'area umida salmastra localizzata sul versante siciliano dello Stretto di Messina (ZPS ITA030042), uno dei bottle-neck lungo la rotta italica di migrazione, dichiarato SIC (ITA 030008) per la sua importanza strategica nell'economia dei flussi migratori dell'avifauna.

Il 27 ottobre 2012 D. Giacobbe e S. Restivo hanno partecipato all'edizione messinese di "Biodiversamente" presso 1'Acquario di Messina, con un intervento mirato ad esporre alle scolaresche l'importanza di tale area per le specie migratorie.

Monitoraggio avifauna acquatica "RNO Laghetti di Marinello". La Riserva Naturale Orientata "Laghetti di Marinello" rappresenta uno dei biotopi di maggiore

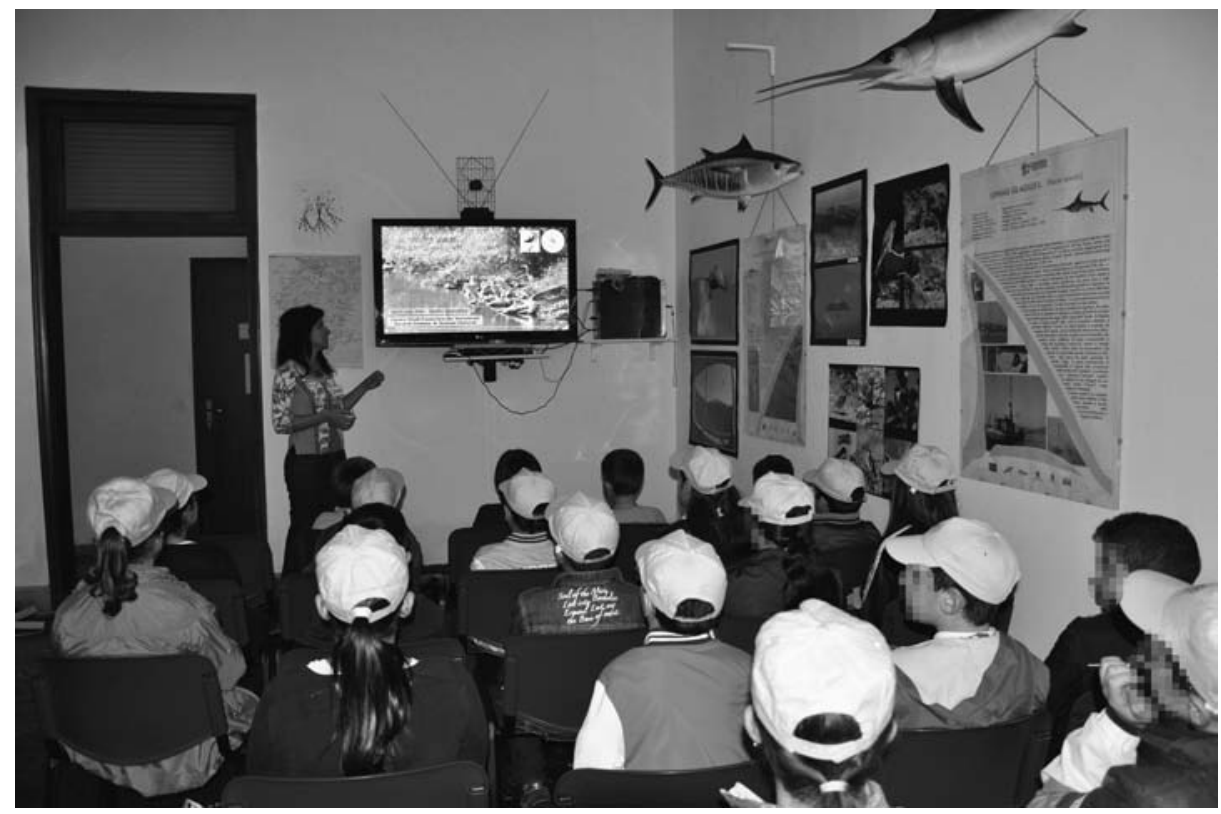

Dalila Giacobbe, relatrice dell'intervento Alla scoperta degli uccelli acquatici della Laguna di Capo Peloro, all'Acquario di Messina per "Biodiversamente 2012". 
interesse del versante costiero settentrionale del Messinese, con spiagge e ambienti umidi utilizzati come luogo di sosta e di svernamento da numerose specie di uccelli acquatici; ciononostante, le informazioni disponibili sull'avifauna acquatica presente nella R.N.O. sono molto scarse. Di conseguenza, nel 2011 Dalila Giacobbe e Salvatore Restivo (CSFV) hanno avviato un progetto a lungo termine di monitoraggio e studio della comunità ornitica acquatica all'interno dell'area protetta.

Lo studio ha già portato alla realizzazione di una tesi di laurea per il Corso triennale in Scienze Naturali dell'Università di Messina, riguardante l'analisi preliminare della scelta dell'habitat da parte dell'avifauna acquatica e l'individuazione di criticità presenti nel sito. I risultati sono stati discussi dal candidato Francesco Rando nella tesi sperimentale "Check-list e distribuzione dell'avifauna acquatica nella Riserva Naturale Orientata "Laghetti di Marinello" (Messina, Sicilia)".

"Studio e valorizzazione didattico-scientifica della collezione naturalistica del Distaccamento Forestale di Colle San Rizzo, Messina". Il progetto CSFV iniziato nel 2011 e condotto da Salvatore Restivo e Dalila Giacobbe in accordo con l'Ispettorato Ripartimentale delle Foreste di Messina, ha lo scopo di valorizzare la collezione, in particolare nella sua funzione didattica, attraverso varie attività quali: l'aggiornamento della nomenclatura sistematica dei reperti, la realizzazione di materiale divulgativo, l'allestimento di mostre tematiche temporanee, l'organizzazione di manifestazioni di divulgazione scientifica ed educazione ambientale.

Quest'anno la collezione è stata oggetto di un contributo scientifico presentato al XXII Congresso dell'Associazione Nazionale Musei Scientifici che si è tenuto a Firenze (Giacobbe \& Restivo, 2012).

Inoltre in occasione della $4^{\mathrm{a}}$ Notte della cultura di Messina la collezione è stata oggetto di una mostra tematica curata dal CSFV dal titolo "La Rete del bracconaggio" insieme ad altri reperti legati al mondo della caccia illegale.

I membri del CSFV Dalila Giacobbe e Salvatore Restivo collaborano col Museo Zoologico "Cambria" dell'Università di Messina alle attività di studio delle collezioni e di divulgazione scientifica. Nel 2012 la collezione mammalogica del Museo è stata oggetto di un contributo scientifico presentato al XXII Congresso dell'Associazione Nazionale Musei Scientifici che si è tenuto a Firenze (Restivo \& Giacobbe, 2012).

Progetto "Ululone", in corso, presso il nostro Centro Studi, da oltre 15 anni (coordinatore: Anna Rita Di Cerbo). Anche quest'anno, è proseguita l'attività di monitoraggio delle popolazioni di Ululone dal ventre giallo (Bombina variegata) in provincia di Bergamo.

Progetto "Nutria" (coordinatori: Roberta Castiglioni e Samuele Venturini). L'attività prosegue, in un'area ristretta, con il progetto sterilizzazione. Dovranno essere messe a punto procedure e protocolli per approfondire gli aspetti di etologia di questa specie alloctona ancora poco conosciuti. È in preparazione un articolo sugli aspetti veterinari dello studio.

Progetto "Avium". (Coordinatore: Stefano Aguzzi). I monitoraggi al Parco di Trenno e alle cave di Muggiano proseguiranno fino al 2014. La pubblicazione dei risultati è prevista per il 2015.

Bibliografia

Di Cerbo A. R. \& Biancardi C. M. 2012 - Are there real sexual morphometric differences in yellow-bellied toads (Bombina spp.; Bombinatoridae)? AmphibiaReptilia, 33 (2): 171-183. DOI: 10.1163/156853812X634062 
Di Cerbo A. R. \& Biancardi C. M. 2012 - Habitat fragmentation and the importance of riparian corridors for mammals: a case study in the Northern Adda Regional Park (SCI IT2030005 Palude di Brivio, Lombardy, Northern Italy). In: VIII Congresso Nazionale di Teriologia (Piacenza 9-11 May 2012). Poster.

Di Cerbo A. R. \& Biancardi C. M. 2012 - Herpetofauna of the Northern Adda Regional Park (SCI IT2030005 Palude Di Brivio, Lombardy, Northern Italy): management and conservation issues. In: IX Congresso Nazionale della Societas Herpetologica Italica (Bari - Conversano, 26-30 September 2012). Poster.

Di Cerbo A. R. \& Biancardi C. M. 2012 - Ecological notes on the Bullfrog Lithobates catesbeianus in an area of the Po plain (Emilia-Romagna, Northern Italy). In: IX Congresso Nazionale della Socìetas Herpetologica Italica (Bari - Conversano, 26-30 September 2012). Poster.

Giacobbe D., Ricciardi D. \& Restivo S. 2012 - Analisi territoriale del rapporto Uomo-Rettile attraverso l'elaborazione dei dati del Centro Recupero Fauna Selvatica di Messina. In: IX Congresso Nazionale della Societas Herpetologica Italica (Bari - Conversano, 26-30 settembre 2012). Poster.

Giacobbe D. \& Restivo S. 2012 - Il ruolo della collezione naturalistica del Distaccamento Forestale di Colle San Rizzo (Messina) nella diffusione della cultura scientifica. In: XXII Congresso Associazione Nazionale Musei Scientifici, Firenze 14-16 novembre 2012. Poster.

Restivo S. Giacobbe D. \& Lo Paro G. 2012 - Attività di studio e valorizzazione della collezione di Mammiferi del Museo Zoologico "Cambrìa" di Messina. In: XXII Congresso Associazione Nazionale Musei Scientifici, Firenze 14-16 novembre 2012. Poster.

\section{Centro Studi Ottimizzazione Biologica}

Coordinatore: Alberto Minetti

Il Centro Studi Ottimizzazione Biologica (CSOB) fu fondato nel 1998 da Alberto Minetti e Franco Saibene (Istituto Tecnologie Biomediche Avanzate del CNR). Una conferenza di McNeill Alexander, Fellow della Royal Society e autoritá universalmente riconosciuta nel campo del movimento umano ed animale, presso il Museo Civico di Storia Naturale costituí nel dicembre 1998 la prima iniziativa del CSOB.

Lo studio dei meccanismi biologici spesso invita i ricercatori a ragionare in termini di ottimizzazione. Comprendere un fenomeno in molti casi coincide con la conoscenza del comportamento del sistema quando le variabili in gioco sono perturbate rispetto ai valori "standard". Configurazioni di valori "standard" possono essere considerate come "ottimizzate" quando una qualsiasi deviazione da esse porta ad un peggioramento in termini di consumo energetico, lavoro meccanico, depauperamento di risorse, aumento della complessità, riduzione dei fattori di sicurezza, e così via.

Ne sono esempi due fenomeni che riguardano la respirazione e la locomozione. Per una certa ventilazione alveolare, e per una data velocità di cammino, esistono frequenze "ideali" di respiro e di passi che minimizzano il dispendio metabolico. Queste frequenze ottimali nascono dal fatto che esiste un compromesso tra lavoro meccanico statico e dinamico (respirazione) e tra lavoro meccanico esterno ed interno (locomozione), tale per cui la loro somma é maggiore per frequenze inferiori o superiori a quella ideale. Il fascino di questi studi nasce anche dal legame 
tra ottimizzazione biologica e selezione evolutiva, laddove specie differenti hanno intrapreso vie alternative per risolvere lo stesso problema funzionale. Ulteriore attrazione è provocata dalle cosiddette "trappole evolutive", situazioni che non rappresentano la soluzione ottimale assoluta di un problema ma che per una determinata specie costituiscono il massimo raggiungibile senza riconfigurare drasticamente la struttura esistente.

Partecipazione a Congressi

VIII Congresso Nazionale di Teriologia, Piacenza 9-11 May 2012. Al Congresso è stata presentata una comunicazione orale dal titolo "Gaits at high speed in free ranging cursorial mammals" (Biancardi \& Minetti, 2012c)

SIF 2012. Società Italiana di Fisiologia. 21-23 Settembre, Verona. Al Congresso sono stati presentati due poster dal titolo: "Skipping' as the gait of choice in hypogravity: metabolic and biomechanical insights from level and gradient experiments on Earth" (Minetti et al., 2012b) e "Optimal interval for periodical lead limb changes during straight gallop in race horses" (Biancardi \& Minetti, 2012b).

Nel corso del 2012 inoltre, il Centro Studi ha proseguito il progetto riguardante lo studio di diversi aspetti della locomozione animale. Dallo studio della locomozione ottapode, una forma di locomozione terrestre comparsa precocemente nel corso dell'evoluzione, caratteristica degli aracnidi, si possono evidenziare analogie e differenze con le andature classiche degli animali bipedi, quadrupedi ed esapodi. Una delle andature quadrupedi, il galoppo, costituisce un secondo filone di ricerca. Questa andatura veloce si manifesta in due forme, galoppo traverso e galoppo rotatorio, i cui determinanti biomeccanici sono oggetto di studio.

Fra i programmi del CSOB per il futuro: cicli di conferenze su argomenti generali, incontri serali periodici, cineteca naturalistica specializzata in locomozione, archivio informatico di pubblicazioni sull'ottimizzazione biologica (consultabile sul sito Internet della SISN), progetti di ricerca multicentrici (multi-museali connessi attraverso la rete), supporto alla didattica museale per la realizzazione di sale e rassegne tematiche. Coinvolgere giovani dottorandi e ricercatori in ricerche miranti ad estendere concetti di ottimizzazione acquisiti nell'uomo ad altre specie, attività che avrebbero come supporto, ad esempio, materiale reperibili in ambito museale. Inoltre, il Centro Studi potrebbe prendersi carico della supervisione di tesi di laurea in Biologia e Medicina.

Grazie al carattere multidisciplinare degli studi di ottimizzazione biologica, il CSOB si relaziona e collabora con gli altri Centri Studi della SISN.

Bibliografia

Biancardi C. M. \& Minetti A.E., 2012 - Biomechanical determinants of transverse and rotary gallop in cursorial mammals. J. Exp. Biol., 215 (23), pp. 4144-4156. Doi: $10.1242 /$ jeb.073031

Biancardi C. M. \& Minetti A.E., 2012b - Optimal interval for periodical lead limb changes during straight gallop in race horses. In: 63 Congresso Nazionale SIF, Verona 21-23 September 2012. Poster.

Biancardi C. M. \& Minetti A.E., 2012c - Gaits at high speed in free ranging cursorial mammals. In: VIII Congresso Nazionale di Teriologia, Piacenza 9-11 May 2012. Oral communication.

Minetti A.E., Pavei G. \& Biancardi C.M., 2012a - The energetics and mechanics of level and gradient skipping: preliminary results for a potential gait of choice in 
low gravity environments., Planetary and Space Science, 74 (1), pp. 142-145. Doi: 10.1016/j.pss.2012.06.004

Minetti A.E., Pavei G. \& Biancardi C.M., 2012b - Skipping as the gait of choice in hypo-gravity: metabolic and biomechanical insights from level and gradient experiments on Earth. In: 63 Congresso Nazionale SIF, Verona 21-23 September 2012. Poster.

Minetti A.E., Pavei G. \& Biancardi C.M., 2012c - Legged locomotion paradigms on Earth can teach humans how to safely extend their progression speed when moving on the Moon. Scientific Preparations for Lunar Exploration workshop, 6 -7 February 2012, ESTEC, Noordwijk, The Netherlands.

\section{Centro Studi Storico Naturalistici}

Coordinatore: Aldo Oriani

Conferenze

Aldo Oriani ha tenuto presso il Museo civico di Scienze naturali "E. Caffi" di Bergamo:

il 9 marzo la conferenza "Appunti sulla fauna dell'Armenia";

il 20 ottobre, nell'ambito di Bergamoscienza 2012, la conferenza "Orso ed uomo: una storia millenaria".

Agnese Visconti, nel2012, ha tenuto corsi per 1'Università della Terza Età organizzati daAUSER sui temi delle risorse energetiche e della storia del paesaggio lombardo. Corradino Guacci, sotto l'egida della Società di storia della fauna, ha tenuto:

il 30 marzo a Campobasso la relazione "Giuseppe Altobello naturalista molisano" nell'ambito del convegno "Giuseppe Altobello naturalista, poeta, medico: l'eclettismo di un molisano"

il 21 aprile a San Giuliano del Sannio presso l'associazione "Insieme" la relazione "Il lupo tra mito e realtà"

il 19 maggio a Pescasseroli nell'ambito del convegno "I novant'anni del Parco: storia e bilanci" la relazione "Conoscenze e politiche faunistiche alle origini del Parco e nella sua prima fase di esistenza"

l'8 agosto 2012 ad Oratino presso l'Associazione culturale A. Giovannitti la relazione "Giuseppe Altobello, medico, naturalista e poeta"

il 13 ottobre a Riccia nell'ambito de "La montagna che era.... a cinquant'anni dal'XI Festa nazionale della Montagna" la relazione "L'uomo e gli animali del bosco";

il 31 ottobre a Roma presso il Museo civico di zoologia, nell'ambito di "M'ammalia la settimana dei Mammiferi" la relazione "Uomini e lupi"

\section{Mostre}

Aldo Oriani, nell'ambito di Bergamoscienza 2012 e sotto l'egida del progetto "Life Arctos" della Regione Lombardia, ha collaborato all'allestimento della mostra "L'orso bruno è tra le nostre montagne .. conosciamolo meglio" tenutasi dal 5 al 21 ottobre presso il Museo civico di Scienze naturali "E. Caffi” di Bergamo.

Bibliografia

Nel corso del 2012 i membri del Centro Studi hanno pubblicato i seguenti lavori: 
Di Bartolo A. \& Visconti A. (a cura di), 2012 - Filippo Parlatore. Viaggio per le parti settentrionali d'Europa fatto nell'anno 1851. Ibis, Como e Pavia.

Oriani F., 2012 - Giuseppe Arrigoni fra rivoluzione e mediazione. In: Atti del Convegno "Dio lo vuole: lecchesi e brianzoli per il risorgimento italiano" Oggiono e Lecco 28-29 ottobre 2011. Archivi di Lecco, Lecco, 35 (1): 70-83.

Visconti A., 2012 - La fondazione dell'Orto botanico di Brera e gli anni della direzione dell'abate vallombrosano Fulgenzio Vitman (1728-1806) tra assolutismo asburgico ed età napoleonica. Atti della Società Italiana di Scienze Naturali e del Museo Civico di Storia Naturale di Milano, Milano, 153 (1): 27-48.

Visconti A., 2012 - Conoscenza e bellezza della natura nel "Bel Paese". In: Un best seller per l'Italia unita "Il Bel Paese" di Antonio Stoppani, P. Redondi (a cura), Città delle Scienze \& Biblioteca Sormani, Milano: 135-169 (www.milanocittadellescienze.it).

\section{Centro Studi Invertebrati}

Coordinatore: Stefano Aguzzi

Il Centro Studi Invertebrati (CSI), nato alla fine del 2011, è un'unità operativa della Società Italiana di Scienze Naturali (SISN) che effettua ricerche sulla biologia e l'ecologia della Fauna Invertebrata terrestre e marina, con particolare riferimento alle specie italiane, e che realizza e promuove iniziative volte alla conservazione di tali specie. Il Centro Studi è aperto a collaborazioni con altri enti, promuove la realizzazione di tesi di Laurea in Scienze Naturali, Biologiche e Ambientali, operando in collaborazione con le università e si propone inoltre di favorire la comunicazione e lo scambio di informazioni ed esperienze tra studiosi ed appassionati.

Durante il suo primo anno il CSI ha effettuato sia attività di ricerca sia di divulgazione scientifica.

Divulgazione scientifica

27 Ottobre: Il CSI ha partecipato alla manifestazione "Immersi nella Biodiversità - dai Monti Peloritani ai fondali dello Stretto di Messina" organizzata dall'Acquario di Messina (CNR - CeSPOM) in collaborazione con Azienda Regionale Foreste Demaniali, Società Italiana di Scienze Naturali e AOM onlus, presso le strutture del'Acquario stesso. I membri Salvatore Restivo e Dalila Giacobbe hanno tenuto una conferenza dal titolo "Lo stagno delle libellule", incentrata sulla biologia e l'ecologia degli Odonati e sulle specie presenti nel "Centro Polifunzionale delle piante endemiche e autoctone del Mediterraneo" sito sui Monti Peloritani (ME). La manifestazione rientra nell'ambito dell'evento nazionale "Biodiversamente" promosso da ANMS e WWF.

Ricerche e progetti

Gli Odonati del "Centro Polifunzionale delle piante endemiche e autoctone del Mediterraneo"

In accordo con l'Ufficio Provinciale di Messina dell'Azienda Foreste Demaniali della Regione Siciliana, il CSI, nelle persone di Salvatore Restivo e Dalila Giacobbe, ha avviato uno studio volto a definire una checklist degli Odonati che frequentano il "Centro Polifunzionale delle piante endemiche e autoctone del Mediterraneo" (Monti Peloritani - Messina) ed in particolare che utilizzano lo stagno 


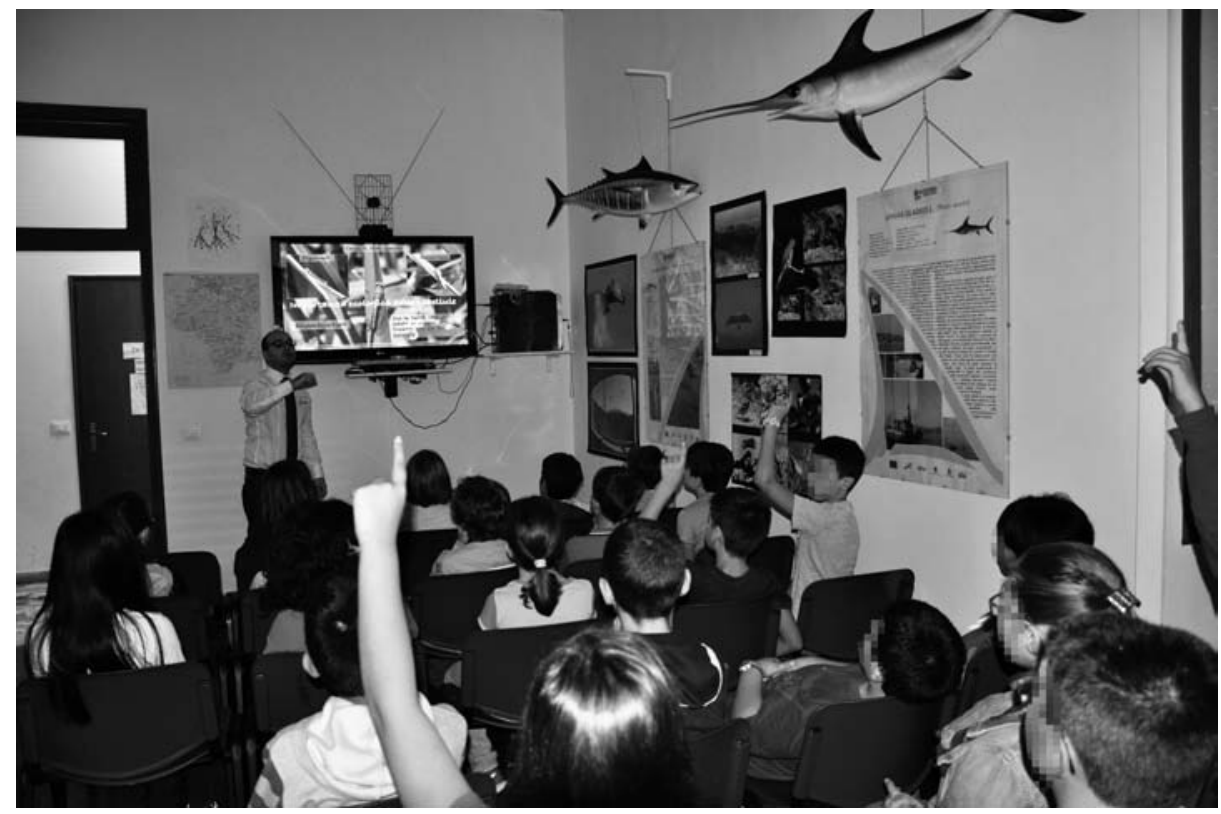

Salvatore Restivo (CSI) risponde alle domande degli studenti durante il seminario "Lo stagno delle libellule", in occasione di "Biodiversamente"( 27 Ottobre 2012, Acquario Civico di Messina).

come sito riproduttivo, raccogliendo inoltre informazioni relative l'eco-etologia delle specie individuate. I dati preliminari raccolti già nel primo anno indicano che lo stagno artificiale del Centro Polifunzionale rappresenta un interessante sito attrattivo per le libellule, oltre che un utile strumento a sostegno delle popolazioni di Odonati nell'area.

\section{Il Granchio di fiume in Sicilia}

Non sono ancora disponibili dati precisi ed aggiornati sullo status e sulla distribuzione generale del Granchio di fiume (Potamon fluviatile) in Sicilia. I dati di presenza già noti per la specie risultano non rappresentativi dell'attuale distribuzione di $P$. fluviatile sull'isola e spesso si riferiscono a segnalazioni risalenti a più di 90 anni fa. Pertanto nel 2006 Salvatore Restivo e Dalila Giacobbe hanno avviato un'indagine a lungo termine sulla presenza di questo Brachiuro nei corsi d'acqua della Sicilia per accertarne la distribuzione attuale, confrontarla con dati storici e raccogliere informazioni di carattere biologico ed ecologico sulle popolazioni individuate. Con la costituzione nel 2011 del CSI, tale ricerca è confluita nei progetti del nuovo Centro Studi. Lo studio sta consentendo di ampliare significativamente l'areale noto per la specie in Sicilia.

Studio della Comunità di Odonati delle Torbiere d'alta quota nelle Province di Bergamo e Sondrio

Il Centro Studi Invertebrati ha svolto una ricerca sulle Comunità di Odonati presenti in alcune torbiere di media e d'alta quota al fine di definire l'odonatofauna presente in ambiente di torbiera, studiare la selezione degli habitat da parte delle 
diverse specie e definire la fenologia delle popolazioni che vivono nei suddetti ambienti. Lo studio ha previsto un censimento qualitativo mediante l'osservazione degli stadi immaginali presso gli habitat acquatici e lungo transetti lineari selezionati nelle aree circostanti. Gli animali di non immediata identificazione sono stati temporaneamente catturati mediante l'utilizzo di retini entomologici. È stato inoltre effettuato un censimento semiquantitativo effettuato mediante conteggio e conservazione di esuvie. La raccolta potrà fornire informazioni oggettive riguardanti la fenologia riproduttiva e l'abbondanza delle differenti specie studiate. In tutti i siti sono stati inoltre rilevati parametri ambientali quali: conducibilità dell'acqua, livello di ossigeno disciolto, $\mathrm{pH}$, profondità degli specchi d'acqua, temperatura dell'acqua e dell'aria, vegetazione acquatica e riparia presente per verificare le eventuali relazioni tra gli stessi e le specie presenti. Sono state censite attualmente 16 specie differenti di libellule (Lestes dryas, Lestes sponsa, Ischnura elegans, Ischnura pumilio, Enallagma cyathigerum, Coenagrion hastulatum, Aeshna cyanea, Aeshna juncea, Anax imperator, Somatochlora alpestris, Somatochlora arctica, Somatochlora metallica, Libellula depressa, Libellula quadrimaculata, Sympetrum danae, Leucorrhinia dubia). Nei siti indagati non è stata rilevata la presenza di specie principalmente planiziali, eccezion fatta per le specie Ischnura elegans, Anax imperator e Somatochlora metallica, quest'ultima rinvenuta solamente presso il sito alla quota inferiore (Lago del Prato, Provincia di Bergamo). Questo studio è stato oggetto di una tesi di laurea magistrale presso l'Università degli Studi di Pavia nell'anno 2013. 